\title{
Selected news items and updates for the practicing clinician
}

\author{
Zachary Mulkey MD
}

- After a recent JAMA meta-analysis regarding the use of hydroxyethyl starch solutions in patients with shock, the FDA is recommending that the volume expander solutions carry a boxed warning for increased risk of kidney injury and death. See a review story here.

- The FDA has expanded the role of the antibiotic telavancin to include nosocomial pneumonia due to $S$ aureus. It was already approved in 2009 for complicated skin \& soft tissue infections. Here is a brief review of the drug.

- Some are calling for the practice of isolating patients colonized with MRSA to stop. This after a study in the NEJM was published showing that daily universal decolonization bathing with chlorhexidine washes was much more effective than active detection/isolation techniques.

- The latest on Sarah Murnaghan, the 10-year-old girl suffering from cystic fibrosis who won a court case allowing her to be placed on the adult lung transplant list.

- I recently viewed a board review DVD that had the expert teacher state their belief that smoking cessation attempts were worthless. Well, a recent Cochrane review seems to say otherwise. Here's the story and review. 